\title{
Phosphonylation and aging mechanisms of mipafox and butyrylcholinesterase: A theoretical study
}

\author{
DING Jun ${ }^{1 \dagger}$, YAO Yuan ${ }^{2 \dagger}$, ZHANG Hui $^{1 *} \&$ Li ZeSheng ${ }^{2,3 *}$ \\ ${ }^{1}$ College of Material Science and Engineering, Harbin University of Science and Technology, Harbin 150080, China; \\ ${ }^{2}$ Academy of Fundamental and Interdisciplinary Sciences, Harbin Institute of Technology, Harbin 150000, China; \\ ${ }^{3}$ Key Laboratory of Cluster Science of Ministry of Education \& School of Chemistry, Beijing Institute of Technology, Beijing 100081, China
}

Received February 20, 2012; accepted March 25, 2012; published online June 15, 2012

\begin{abstract}
Phosphonylation and aging processes between butyrylcholinesterase with mipafox have been studied at the B3LYP/6-311G(d,p) level of theory. The calculated results indicate that the phosphonylation process employs a two-step addition-elimination mechanism with the addition (the first step) as the rate-limiting step. Two different calculation models revealed that the catalytic triad of butyrylcholinesterase plays an important role in accelerating the reaction. This is the same mechanism as the phosphonylation reaction of acetylcholinesterase by sarin reported by Wang et al. However, the energy barrier of the rate-limiting step in the present reaction is higher than that in phosphonylation reaction of acetylcholinesterase by sarin. This indicates the differences in the phosphonylation activity of sarin and mipafox. The aging process occurs through a two-step addition-elimination mechanism similar to the phosphonylation process with the addition as the rate-limiting step. The solvent effects have been evaluated by using a CPCM model and the results show that the stationary structures and the negative charges around some important atoms involved in the two processes are not significantly different. However, the energy barrier of the phosphonylation process is remarkably decreased, revealing that this process is feasible in solution.
\end{abstract}

acetylcholinesterase, butyrylcholinesterase, quantum chemical calculations, reaction mechanism, solvent effect

Citation: Ding J, Yao Y, Zhang H, et al. Phosphonylation and aging mechanisms of mipafox and butyrylcholinesterase: A theoretical study. Chin Sci Bull, 2012, 57: 4453-4461, doi: 10.1007/s11434-012-5280-2

Cholinesterases are classified into two major subfamilies based on their substrate and inhibitor specificities: acetylcholinesterase (AChE; EC 3.1.1.7) and butyrylcholinesterase (BChE; EC 3.1.1.8). AChE, a serine hydrolase, is a membrane bound enzyme present in cholinergic neurons which plays an essential role in the central and peripheral nervous systems [1,2]. Massoulie et al. [3] reported that $\mathrm{AChE}$ can hydrolyze acetylcholine released at the synaptic cleft and the neuromuscular junction in response to nerve action potential. In contrast to $\mathrm{AChE}$, the physiological function of $\mathrm{BChE}$ is unclear [4,5]. Despite having no identified endogenous substrate, BChE can efficiently degrade succinylcholine and cocaine and plays an important role in

$\dagger$ These authors contributed equally to this work.

*Corresponding authors (email: hust_zhanghui11@ @otmail.com; zeshengli@bit.edu.cn) detoxification [6]. The X-ray crystal structures of AChE [7] and BChE [8] indicate that they have amino acid sequence homology of $65 \%$ and amino acid sequence identity of $54 \%$. The same Ser-His-Glu catalytic triad is found at the bottom of a deep gorge in both AChE [9] and BChE [8].

As one of the groups of inhibitors of cholinesterase, organophosphorus (OP) compounds are used as pesticides in agriculture, as antihelminthics in medical practice, as additives to hydraulic fluid and jet engine oil in the airline industry, and as chemical warfare agents [10]. Moreover, cholinesterase inhibitors have been also confirmed to play an important role in the treatment of Alzheimer's disease (AD). They can inhibit both AChE and BChE by phosphonylation of the active site serine residue. The hydroxyl oxygen of the active site serine attacks the phosphorus atom of an OP compound to displace the primary leaving group and form 
the OP adduct through a covalent bond. Although it remains unclear whether phosphonylated $\mathrm{BChE}$ can result in toxicity directly [11], this process is of considerable toxicological interest. Kropp and Richardson [12] reported that the extent of inhibition and the chemical composition of the OP adduct can change with time depending on both the rate of reactivation and the rate and nature of aging. Therefore, investigation of the mechanisms of inhibition and aging of both $\mathrm{AChE}$ and $\mathrm{BChE}$ by OP compounds are important for accurately predicting the conjugates formed on both $\mathrm{AChE}$ and $\mathrm{BChE}$ resulting from OP compound exposure, developing biomarkers of exposure to these agents and understanding toxicity. Kropp and Richardson [12,13] reported the products of both inhibition and aging processes between cholinesterases and the organophosphorus compound mipafox. As shown in Scheme 1, both AChE and BChE form similar conjugates with mipafox. Then, the aging of the mipafox$\mathrm{BChE}$ conjugate is initiated by water followed by release of one of the isopropylamine groups. In contrast, the mipafox$\mathrm{AChE}$ conjugate releases both isopropylamine groups during the aging process. However, to date, the fundamental reaction mechanisms for both the phosphonylation and aging processes are not defined.

The studies of the enzymatic reaction mechanisms of AChE [14] and BChE $[6,15]$ show that serine and histidine act as the nucleophilic attacking group and general acid-base catalytic elements, respectively, and the reaction is facilitated by simultaneous proton transfer from serine to histidine. Wang et al. reported the phosphonylation mechanism of sarin and $\mathrm{AChE}$ [16] and the oxime-induced reactivation of sarin-inhibited AChE [17] using density functional theory (DFT). However, no reports have been published on the reaction mechanism between $\mathrm{BChE}$ and its inhibitors based on quantum chemical calculations. In the present study, we constructed a methanol model as a representation of the catalytic serine of $\mathrm{BChE}$ to theoretically explore the phosphonylation and aging mechanisms between $\mathrm{BChE}$ and mipafox by density functional theory. The effect of aqueous solvation was considered via the CPCM model at the same calculation level to evaluate the solvent influences on the reactions.

\section{Computational details}

All quantum chemical calculations were performed with the Gaussian 03 program package [18]. The density functional theory (DFT) with Becke's three-parameter (B3) [19] exchange functional along with the Lee-Yang-Parr (LYP) $[20,21]$ nonlocal correlation functional (B3LYP) was applied in the present study, and the standard valence triple- $\zeta$ basis set, augmented with d-type polarization functions for heavy elements and p-type polarization functions for $\mathrm{H}$, 6-311G(d,p) [22], was used. All of the calculation models were fully optimized by analytic gradient techniques. Harmonic vibrational frequencies were calculated to check whether the obtained species is minima (with all real frequencies) or transition state (with one and only one imaginary frequency). For each transition state, the intrinsic reaction coordinate (IRC) calculations were performed to guarantee its correct connection to the minima. To evaluate the solvation effects on the reactions, the CPCM model with a dielectric constant $\varepsilon=78.39$ was employed to perform the structure optimization and IRC calculation at the B3LYP/6$311 \mathrm{G}(\mathrm{d}, \mathrm{p})$ level. In the CPCM model, the electrostatic interactions between the charges on the solute molecules and the induced charges on the polarizable solvent molecules are solved self-consistently and a simple scaled conductor boundary condition is used which improves the precision and the efficiency [23-26]. All Gibbs free energies were calculated at room temperature and $1 \mathrm{~atm}(1 \mathrm{~atm}=1.01 \times$ $10^{5} \mathrm{~Pa}$ ) pressure. Natural Population Atomic (NPA) charges

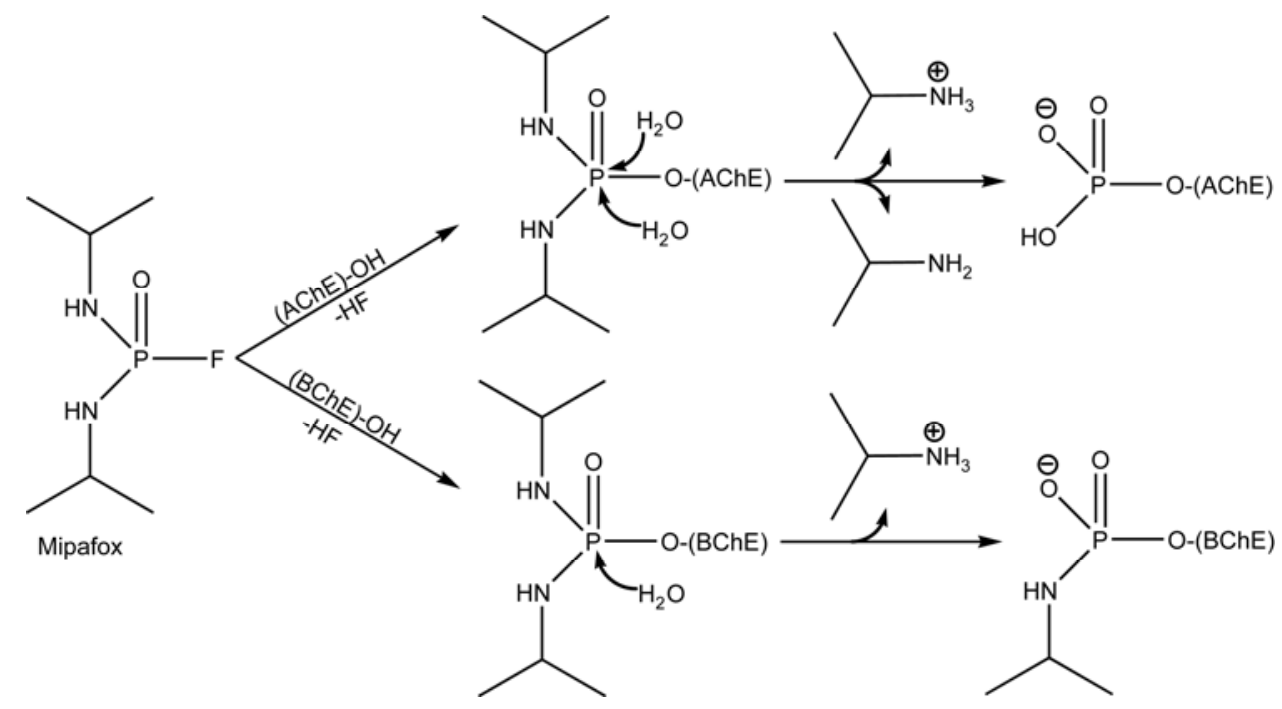

Scheme 1 Pathways of inhibition and aging of mipafox for AChE and BChE. 
were determined at the same level with the Natural Bond Order (NBO) analysis [27-30]. In this paper, all energies mentioned are the Gibbs free energies. In order to simply our discussion, the energies of reactants are set to be zero for reference purposes.

\section{Results and discussion}

The phosphonylation of BChE by mipafox occurs by nucleophilic attack on the phosphorus atom by the hydroxyl oxygen atom in the catalytic serine, displacing the primary leaving group, fluoride, and generating the same $N, N^{\prime}$ diisopropylphosphorodiamido adduct. Then, the adduct undergoes the aging process to eliminate an isopropylamine group [12]. It is difficult to investigate these reaction mechanisms using DFT theory if the whole enzyme system is considered, thus, for a simplified model, methanol $\mathrm{CH}_{3} \mathrm{OH}$ was chosen to represent the active site serine in the enzyme. This enzyme model was also applied in the studies of the reaction mechanism between sarin and AChE by Wang et al. [16].

\subsection{Phosphonylation mechanism}

The nucleophilicity of the catalytic serine in the phospho- nylation reaction can be enhanced by proton transfer to the imidazole nitrogen of the catalytic histidine residue in the catalytic triad. Thus, both the deprotonated $\mathrm{CH}_{3} \mathrm{O}^{-}$anion and neutral $\mathrm{CH}_{3} \mathrm{OH}$ were considered to explore the catalytic effect of the catalytic triad.

\subsection{Pathway A}

Pathway A describes the reaction between $\mathrm{CH}_{3} \mathrm{O}^{-}$and mipafox when the proton in the hydroxyl group of the serine residue has absolutely transferred to the imidazole nitrogen of the histidine residue in the catalytic triad. The structures of reactant, intermediate, transition states and product are shown in Figure 1. The transition states are determined with only one imaginary frequency and IRC calculations were performed to confirm that the transition states connect the corresponding minima. The profile of pathway A is shown in Figure 2. The NPA charges of several important atoms by NBO analysis are listed in Table 1.

Anionic $\mathrm{CH}_{3} \mathrm{O}^{-}$and mipafox, which form a stable complex by two hydrogen bonds in the reactant $\mathrm{Ra}$, generate a covalent intermediate Ia through the nucleophilic attack. Subsequently, the P-F bond is broken, resulting in the generation of the final product $\mathrm{Pa}$. Obviously, this is a two-step addition-elimination reaction.
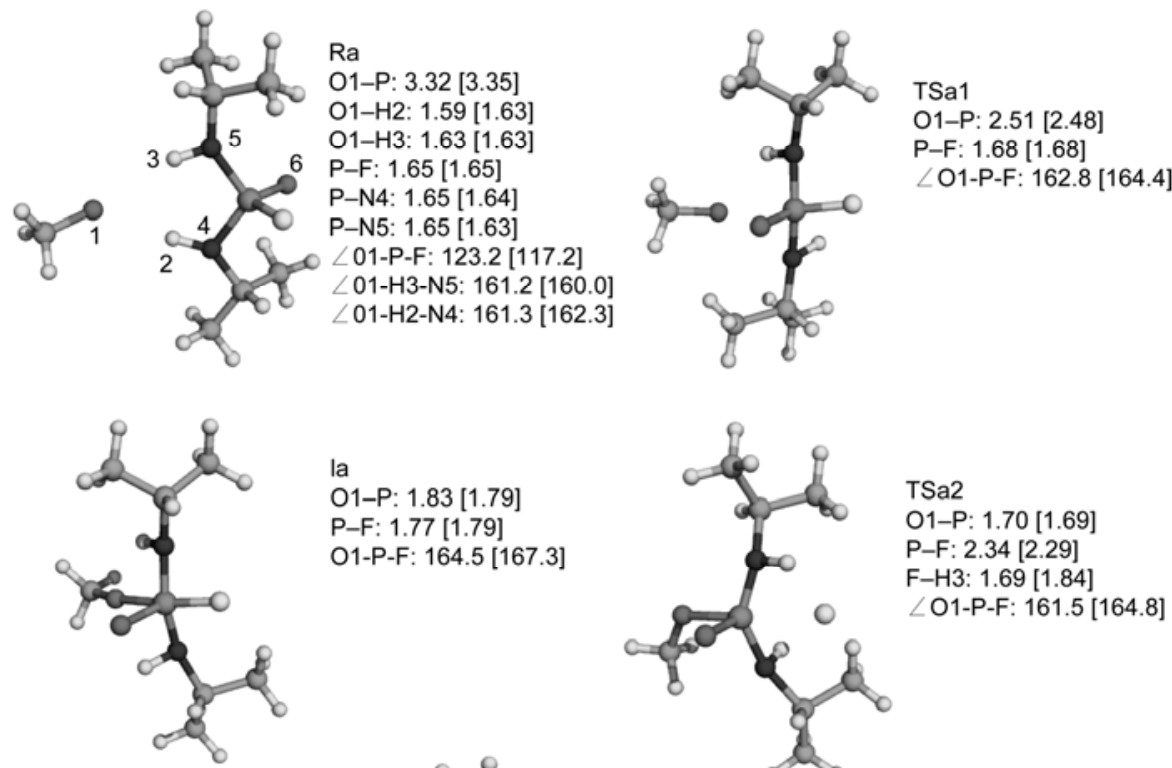

P-F: $1.77[1.79]$

O1-P-F: 164.5 [167.3]

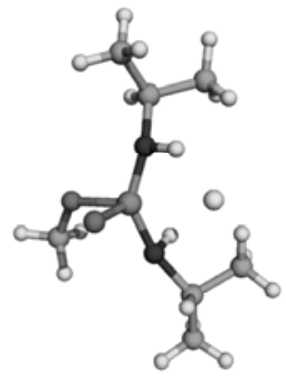

TSa2

O1-P: 1.70 [1.69]

P-F: 2.34 [2.29]

F-H3: 1.69 [1.84]

$\angle$ O1-P-F: 161.5 [164.8]

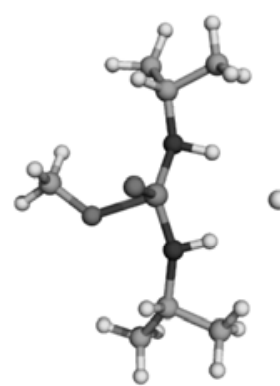

$\mathrm{Pa}$

O1-P: 1.66 [1.64]

P-F: 3.26 [3.29]

$\mathrm{F}-\mathrm{H} 2: 1.62[1.64]$

$\mathrm{F}-\mathrm{H} 3: 1.56[1.72]$

$\angle$ F-N2-N4: 155.5 [159.5]

$\angle$ F-H3-N5: 160.9 [156.1]

Figure 1 Optimized structures of the phosphonylation reaction for pathway A. The values out of and in square brackets are for the gas phase and solvent phase, respectively. Distances are in angstroms and atomic angles are in degrees. 


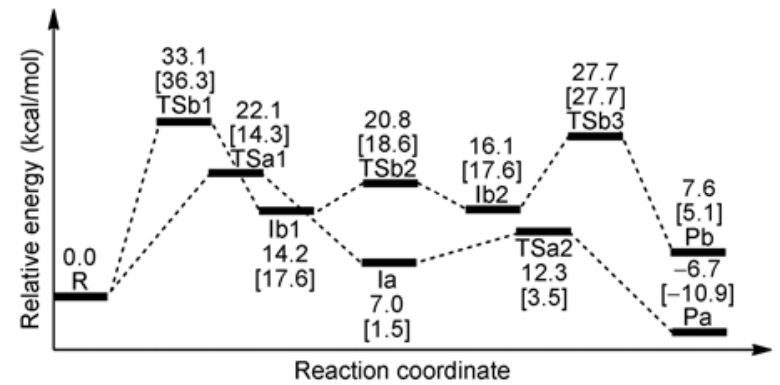

Figure 2 The potential energy surfaces of pathways A and B for the phosphonylation reaction. The values out of and in square brackets are for the gas phase and solvent phase, respectively.

In $\mathrm{Ra}$, weak hydrogen-bond interactions are formed between the $\mathrm{O} 1$ atom of anionic $\mathrm{CH}_{3} \mathrm{O}^{-}$and the $\mathrm{H} 2$ and $\mathrm{H} 3$ atoms in the two amino-group of mipafox to stabilize the two separated reactants. The hydrogen bond lengths are 1.63 and $1.59 \AA$, respectively, and the bond angles are $161.3^{\circ}$ and $161.2^{\circ}$, respectively. The atomic distance between $\mathrm{O} 1$ and $\mathrm{P}$ is $3.32 \AA$, which indicates there is no interaction between the two separated species.

The transition state corresponding to serine's nucleophilic attack, TSa1, has only one imaginary frequency of $173 \mathrm{i} \mathrm{cm}^{-1}$. The vibrational mode corresponding to the imaginary frequency indicates the direct nucleophilic attack on the $\mathrm{P}$ atom of mipafox by $\mathrm{O} 1$ of the anionic $\mathrm{CH}_{3} \mathrm{O}^{-}$from the backside of the $\mathrm{F}$ atom. The angle of the O1-P-F atoms is $162.8^{\circ}$, indicating an almost linear attack. The distance between the $\mathrm{O} 1$ and $\mathrm{P}$ atoms in TSa1 is $2.51 \AA$, about $0.81 \AA$ shorter than that in $\mathrm{Ra}$. The bond length of the $\mathrm{P}-\mathrm{F}$ bond increases from $1.64 \AA$ in Ra to $1.68 \AA$ in TSa1, suggesting its weakening. The NPA charges determined at the same theoretical level show the $\mathrm{O} 1$ atom in anionic $\mathrm{CH}_{3} \mathrm{O}^{-}$has strong nucleophilic character with a negative charge of $-0.66 \mathrm{au}$. The Gibbs free energy barrier of this step is 22.1 $\mathrm{kcal} / \mathrm{mol}$ at $298 \mathrm{~K}$ and $1 \mathrm{~atm}$ pressure.

The intermediate Ia, with a trigonal bipyramidal structure, is formed in this pathway. It lies $7.0 \mathrm{kcal} / \mathrm{mol}$ above the reactant $\mathrm{Ra}$, indicating its lower stabilization than $\mathrm{Ra}$. The O1-P bond is formed with a distance of $1.83 \AA$ and the bond length of $\mathrm{P}-\mathrm{F}$ increases from $1.68 \AA$ in TSa1 to $1.77 \AA$ in Ia.

The transition state $\mathrm{TSa} 2$ has only one imaginary frequency, $160 \mathrm{i} \mathrm{cm}^{-1}$, and connects Ia and the product. The vibrational mode corresponding to the imaginary frequency shows that the $\mathrm{F}$ atom is away from the $\mathrm{P}$ atom and is surrounded by two side groups of mipafox. In TSa2, the P-F bond is broken and the atomic distance is $2.34 \AA$. The $\mathrm{P}-\mathrm{O}$ bond is strengthened because its bond length is shortened to $1.70 \AA$. The NPA charge indicates that the negative charge distributed around the $\mathrm{F}$ atom increases to -0.63 au $(-0.51$ au in Ia and -0.44 au in TSa1) and the negative charge distributed around the $\mathrm{O} 1$ atom decreases to -0.57 au $(-0.63$ au in Ia and -0.66 au in TSa1). The Gibbs free energy barrier of this step is $5.3 \mathrm{kcal} / \mathrm{mol}$ at $298 \mathrm{~K}$ and $1 \mathrm{~atm}$ pressure.

In $\mathrm{Pa}$, the $\mathrm{F}$ atom has strong interactions with mipafox through two hydrogen bonds between the $\mathrm{F}$ anion and the two amino-groups in mipafox with hydrogen bonds lengths of 1.62 and $1.56 \AA$, respectively, and bond angles of $155.5^{\circ}$ and $160.9^{\circ}$, respectively. The atomic distance between $\mathrm{P}$ and $\mathrm{F}$ is $3.26 \AA$, meaning they no longer interact. The NPA charge indicates that the negative charge distributed around the $\mathrm{F}$ atom is $-0.64 \mathrm{au}$. The energy of $\mathrm{Pa}$ is $6.7 \mathrm{kcal} / \mathrm{mol}$ lower than that in $\mathrm{Ra}$, revealing that the product is more thermodynamically stable than $\mathrm{Ra}$ and the reaction along this pathway is exothermic. Pa might be detected by experiments.

To evaluate the solvent effects on this reaction pathway, the structures of the reactant, the transition states, the intermediate and the product were optimized with the CPCM

Table 1 NPA charges of several important atoms involved in phosphonylation reaction by NBO analysis ${ }^{\text {a) }}$

\begin{tabular}{|c|c|c|c|c|}
\hline & $\mathrm{O} 1$ & O6 & $\mathrm{P}$ & $\mathrm{F}$ \\
\hline $\mathrm{Ra}$ & $-0.65[-0.66]$ & $-0.65[-0.65]$ & $1.26[1.27]$ & $-0.39[-0.40]$ \\
\hline TSa1 & $-0.66[-0.67]$ & $-0.62[-0.63]$ & $1.29[1.30]$ & $-0.44[-0.44]$ \\
\hline Ia & $-0.63[-0.62]$ & $-0.64[-0.66]$ & $1.31[1.31]$ & $-0.51[-0.51]$ \\
\hline TSa2 & $-0.57[-0.57]$ & $-0.62[-0.63]$ & $1.27[1.29]$ & $-0.63[-0.63]$ \\
\hline $\mathrm{Pa}$ & $-0.53[-0.53]$ & $-0.62[-0.63]$ & $1.20[1.20]$ & $-0.64[-0.66]$ \\
\hline $\mathrm{Rb}$ & $-0.45[-0.43]$ & $-0.62[-0.62]$ & $1.31[1.31]$ & $-0.35[-0.36]$ \\
\hline TSb1 & $-0.64[-0.63]$ & $-0.54[-0.52]$ & $1.40[1.39]$ & $-0.38[-0.41]$ \\
\hline $\mathrm{Ib} 1$ & $-0.62[-0.61]$ & $-0.49[-0.50]$ & $1.45[1.45]$ & $-0.43[-0.44]$ \\
\hline TSb2 & $-0.56[-0.56]$ & $-0.49[-0.49]$ & $1.40[1.43]$ & $-0.45[-0.48]$ \\
\hline $\mathrm{Ib} 2$ & $-0.55[-0.55]$ & $-0.48[-0.49]$ & $1.43[1.43]$ & $-0.48[-0.48]$ \\
\hline TSb3 & $-0.51[-0.52]$ & $-0.48[-0.48]$ & $1.36[1.37]$ & $-0.58[-0.59]$ \\
\hline $\mathrm{Pb}$ & $-0.50[-0.50]$ & $-0.64[-0.65]$ & $1.27[1.27]$ & $-0.35[-0.39]$ \\
\hline
\end{tabular}

a) The values (au) out of and in square brackets are got in gas phase and in solvent phase, respectively. 
water model and the results are plotted in Figure 1. The NPA charges of several important atoms involved in solvent phase structures by NBO analysis are listed in Table 1 and the profile of this reaction pathway is also shown in Figure 2. By comparing with the results in the gas phase, one can see that the reaction pathway in the solvent phase is very similar to that in the gas phase. However, the conformations of all stationary points and the NPA charges of several important atoms have been slightly changed. The remarkable difference of the reactions in the gas and solvent phases is that the free energy barrier of the rate-limiting step is decreased to $14.3 \mathrm{kcal} / \mathrm{mol}$. Moreover, the whole reaction is exothermic by $10.9 \mathrm{kcal} / \mathrm{mol}$ in the solvent phase, which is
$4.2 \mathrm{kcal} / \mathrm{mol}$ higher than that in the gas phase $(6.7 \mathrm{kcal} / \mathrm{mol})$. These changes might be caused by the structure differences in the gas phase and solvent phase. It can be concluded that the solvent effect can increase the rate of the entire phosphonylation reaction.

\subsection{Pathway B}

Pathway B describes the phosphonylation reaction between neutral $\mathrm{CH}_{3} \mathrm{OH}$ and mipafox. The structures of the reactant, intermediates, transition states, and product are shown in Figure 3. The transition states are determined with only one imaginary frequency and IRC calculations were carried out
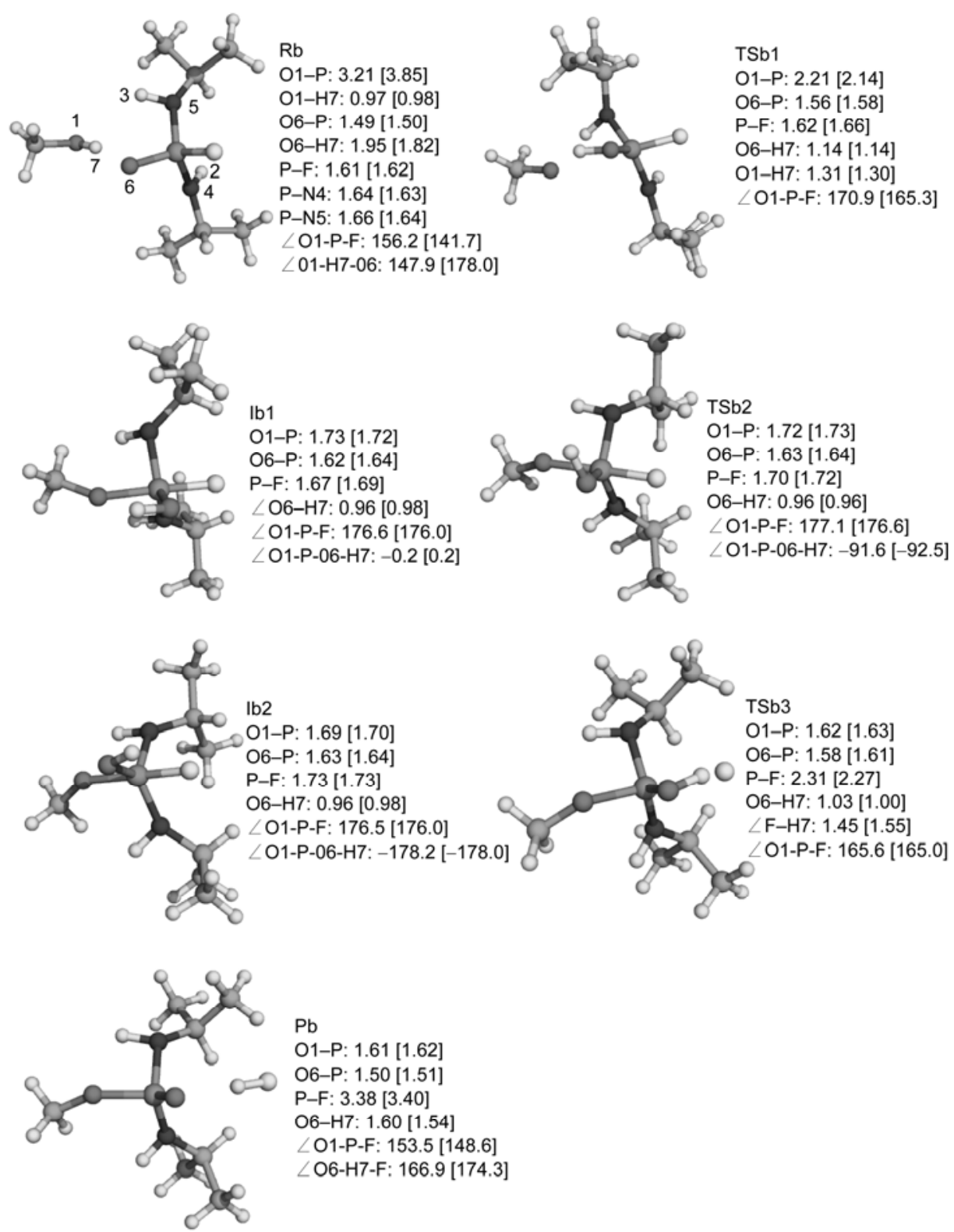

$\mathrm{Pb}$

O1-P: $1.61[1.62]$

O6-P: 1.50 [1.51]

$\mathrm{P}-\mathrm{F}: 3.38[3.40]$

O6-H7: $1.60[1.54]$

$\angle O 1-P-F: 153.5[148.6]$

$\angle \mathrm{O} 6-\mathrm{H} 7-\mathrm{F}: 166.9[174.3]$

Figure 3 Optimized structures of the phosphonylation reaction for pathway B. The values out of and in square brackets are for the gas phase and solvent phase, respectively. Distances are in angstroms and atomic angles are in degree. 
to confirm that the transition states connect the corresponding minima. The NPA charges of several important atoms involved in this pathway by NBO analysis are listed in Table 1. The profile of pathway $\mathrm{B}$ presented in Figure 2 indicates that pathway $\mathrm{B}$ is also a two-step addition-elimination reaction mechanism, similar to pathway $\mathrm{A}$.

The neutral $\mathrm{CH}_{3} \mathrm{OH}$ forms a stable complex, $\mathrm{Rb}$, with mipafox by a hydrogen bond between the $\mathrm{H} 7$ atom of the hydroxyl group in $\mathrm{CH}_{3} \mathrm{OH}$ and the $\mathrm{O} 6$ atom in mipafox. The bond length is $1.95 \AA$ and the bond angle is $147.9^{\circ}$. The distance between the $\mathrm{P}$ and $\mathrm{O} 1$ atoms is $3.21 \AA$ and the length of the P-F bond is $1.61 \AA$.

The transition state TSb1 is determined by only one imaginary frequency of $990 \mathrm{i} \mathrm{cm}{ }^{-1}$. The vibrational mode corresponding to the imaginary frequency indicates that the nucleophilic attack on the $\mathrm{P}$ atom in mipafox by the $\mathrm{O} 1$ atom occurs while the $\mathrm{H} 7$ proton of the hydroxyl group in $\mathrm{CH}_{3} \mathrm{OH}$ is transferred to the $\mathrm{O} 6$ atom in mipafox. The atomic distance between $\mathrm{O} 1$ and $\mathrm{H} 7$ is $1.31 \AA$ and the bond length of $\mathrm{O} 6-\mathrm{H} 7$ is $1.14 \AA$. The atomic angle of O1-P-F is $170.9^{\circ}$, which supports the linear nucleophilic attack. The atomic distance between the $\mathrm{O} 1$ atom of the hydroxyl group in $\mathrm{CH}_{3} \mathrm{OH}$ and the $\mathrm{P}$ atom in mipafox is $2.21 \AA$, which is $0.30 \AA$ shorter than that in TSa1. The bond length of P-F is $1.62 \AA$, which is $0.08 \AA$ shorter than that in TSa1. The NPA charges display a -0.64 au negative charge around the $\mathrm{O} 1$ atom of serine, which is 0.02 au less than that in TSa1. This change means that the nucleophilic ability of the $\mathrm{O} 1$ atom of serine in TSal is stronger than that in TSb1. The bond length of P-O6 is increased from $1.49 \AA$ in $\mathrm{Rb}$ to $1.56 \AA$ in TSb1. The Gibbs free energy barrier of this step is 33.1 $\mathrm{kcal} / \mathrm{mol}$ at $298 \mathrm{~K}$ and $1 \mathrm{~atm}$ pressure, which is $11.0 \mathrm{kcal} / \mathrm{mol}$ higher than that in pathway $\mathrm{A}$ in the gas phase.

The trigonal bipyramidal intermediate Ib1, similar to Ia, lies $14.2 \mathrm{kcal} / \mathrm{mol}$ above $\mathrm{Rb}$, indicating weak stability. The $\mathrm{P}-\mathrm{O} 6$ bond length is $1.62 \AA$, which indicates that the double bond $\mathrm{P}-\mathrm{O} 6$ in $\mathrm{Rb}$ converts to a single bond with $\mathrm{H} 7$ transfer. The NPA charge shows that the negative charge of $\mathrm{O} 1$ is increased from $-0.45 \mathrm{au}$ in $\mathrm{Rb}$ to -0.62 au in $\mathrm{Ib} 1$ and the negative charge of $\mathrm{O} 6$ is decreased from $-0.62 \mathrm{au}$ in $\mathrm{Rb}$ to -0.49 au in Ib1. This change in charge confirms that the $\mathrm{H} 7$ transfer is a proton transfer. The bond length of the $\mathrm{P}-\mathrm{O} 1$ bond is $1.73 \AA$ and the bond length of the $\mathrm{P}-\mathrm{F}$ bond is weakened to $1.67 \AA$ from $1.62 \AA$ in TSb1.

Connecting to $\mathrm{Ib} 1$, the transition state $\mathrm{TSb} 2$ characterizes the rotation of the $\mathrm{H} 7$ atom from the side of the $\mathrm{O} 1$ atom to the opposite side where the rotated $\mathrm{H} 7$ atom is close to the $\mathrm{F}$ atom by the analysis of the vibrational mode. The only imaginary frequency of TSb2 is $354 \mathrm{icm}^{-1}$. The dihedral angle of O1-P-O6-H7 in TSb2 is rotated to $-91.6^{\circ}$ from $-0.2^{\circ}$ in $\mathrm{Ib} 1$. The bond distances of the $\mathrm{P}-\mathrm{F}$ bond and $\mathrm{P}-\mathrm{O} 1$ bond are 1.70 and $1.72 \AA$, respectively. The Gibbs free energy barrier of this rotation process is $6.6 \mathrm{kcal} / \mathrm{mol}$ at $298 \mathrm{~K}$ and $1 \mathrm{~atm}$ pressure.

The intermediate $\mathrm{Ib} 2$, which has the same trigonal bi- pyramidal structure as $\mathrm{Ib} 1$, is calculated to be $4.7 \mathrm{kcal} / \mathrm{mol}$ below TSb2. The dihedral angle of O1-P-O6-H7 is $-178.2^{\circ}$. The bond length of the P-O1 bond is shortened to $1.69 \AA$ and the P-F bond length is stretched to $1.73 \AA$. This indicates that the $\mathrm{P}-\mathrm{F}$ bond has become weaker.

The transition state TSb3 characterizes the P-F bond fission and $\mathrm{F}$ anion departure process for the elimination step. The one imaginary frequency is $273 \mathrm{i} \mathrm{cm}^{-1}$ and the vibrational mode reveals that the leaving $\mathrm{F}$ atom is surrounded by certain $\mathrm{H}$ atoms in mipafox. The atomic distance between $\mathrm{F}$ and $\mathrm{H} 7$ is $1.45 \AA$; however, there is no hydrogen bond formed between them. The $\mathrm{P}-\mathrm{O} 1$ bond length is shortened to $1.62 \AA$ and the atomic distance between the $\mathrm{P}$ and $\mathrm{F}$ atoms is calculated to be $2.31 \AA$. The atomic angle of O1-P-F is $165.6^{\circ}$, indicating that these atoms are almost maintained in a linear conformation. The Gibbs free energy barrier of this step is $11.6 \mathrm{kcal} / \mathrm{mol}$.

The energy of $\mathrm{Pb}$ is $20.1 \mathrm{kcal} / \mathrm{mol}$ lower than that of $\mathrm{TSb} 3$, indicating that it is very feasible energetically to form $\mathrm{Pb} . \mathrm{Pb}$ is a stable complex because of the hydrogen bond formed between the $\mathrm{H} 7$ in the HF molecule and the $\mathrm{O} 6$ in mipafox. The hydrogen bond length and angle are $1.60 \AA$ and $166.9^{\circ}$, respectively. The NPA charge shows that the negative charge of $\mathrm{O} 6$ is increased from -0.48 au in TSb3 to $-0.64 \mathrm{au}$ in $\mathrm{Pb}$ and the negative charge of $\mathrm{F}$ is decreased from -0.58 au in TSb3 to -0.35 au in $\mathrm{Pb}$, indicating the occurrence of the $\mathrm{H} 7$ proton transfer. The $\mathrm{P}-\mathrm{O} 6$ bond length of $1.50 \AA$ in $\mathrm{Pb}$ reveals that the $\mathrm{P}-\mathrm{O} 6$ bond is converted into a double bond from a single bond in $\mathrm{Ib} 2$ with a bond length of $1.63 \AA$. $\mathrm{Pb}$ lies $7.6 \mathrm{kcal} / \mathrm{mol}$ above $\mathrm{Rb}$, which indicates that this reaction along Pathway $\mathrm{B}$ is thermodynamically endothermal.

To evaluate the solvent effects on this reaction pathway, the structures of reactant, transition states, intermediate, and product were optimized using the CPCM water model and the results are shown in Figure 3. The NPA charges of several important atoms involved in solvent phase structures by NBO analysis are listed in Table 1 and the profile of this pathway is shown in Figure 2. It is evident that the solvent phase pathway has similar characteristics as the gas phase pathway. However, the structures of the stationary points and the NPA charges of several important atoms involved in the solvent phase differ by comparison with the relative structures in the gas phase. The free energy barrier of the rate-limiting step in the solvent phase is $36.3 \mathrm{kcal} / \mathrm{mol}$, which is $3.2 \mathrm{kcal} / \mathrm{mol}$ higher than that in gas phase.

Both pathways A and B employ a two-step additionelimination mechanism with the addition process (the first nucleophilic attack step) as the rate-limiting step. The major products in both pathways are consistent with the experimental results [12]. However, the free energy barriers of the rate-limiting step in Pathway $\mathrm{A}$ in both the gas phase and the solvent phase are lower than those in Pathway B. This indicates that the phosphonylation reaction along pathway $\mathrm{A}$ is more feasible. The differences in the free energy barri- 
ers between the two pathways also prove that the catalytic triad of BChE can effectively accelerate the phosphonylation process by accepting the proton from the serine residue as it does in the acylation reaction.

In both the present study and the reported phosphonylation mechanism of sarin and AChE by Wang et al. [16], the same calculation level is employed and a similar methanol $\mathrm{CH}_{3} \mathrm{OH}$ model is chosen to represent the active site serine in both enzymes. Both sarin and mipafox are organophosphorus (OP) compounds. Therefore, it is reasonable to examine the phosphonylation mechanisms of cholinesterase by organophosphorus compounds by comparing both research results. One can see that both cholinesterases employ the same two-step addition-elimination reaction mechanism with the addition as the rate-limiting step. In the two different reaction pathways in the present study, the negative charges around the $\mathrm{O} 1$ atom of the serine residue at the active site are less than that in the phosphonylation reaction of sarin and AChE by Wang et al. [16], which indicates that the latter has stronger nucleophilicity than the former. This results in the Gibbs free energy barriers of the present reaction being higher than those in the phosphonylation reaction of sarin and AChE by Wang et al. [16].

\subsection{Aging mechanism}

A phosphonylated enzyme may undergo aging by water to generate a negatively charged product. In order to understand toxicity and develop biomarkers for determining exposure to these agents, we have theoretically studied the aging mechanism using a calculation model containing the mipafox-enzyme conjugate (the major product of pathway A and pathway B) and a water molecule.

The structures of reactant, intermediate, transition states and the product and the energy profile are shown in Figure 4. In this reaction, $\mathrm{H}_{2} \mathrm{O}$, which is $\mathrm{H}$-bonded to the mipafoxenzyme conjugate in the reactant (Rc), attacks the $\mathrm{P}$ atom of the mipafox-enzyme conjugate and forms an intermediate product (Ic), while $\mathrm{H} 9$ in $\mathrm{H}_{2} \mathrm{O}$ is transferred to the $\mathrm{N} 4$ atom of the mipafox-enzyme conjugate. Subsequently, a single

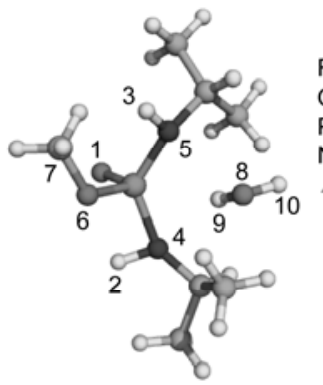

8-P: 3.92 [4.00] P-N4: 1.71 [1.69] N4-H9: 2.02 [2.11] ZO8-H9-N4: 167.0 [165.4]

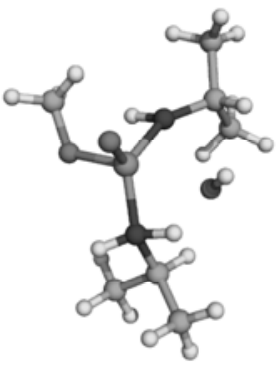

O8-P: 2.14 [2.25]

P-N4: 1.84 [1.83]

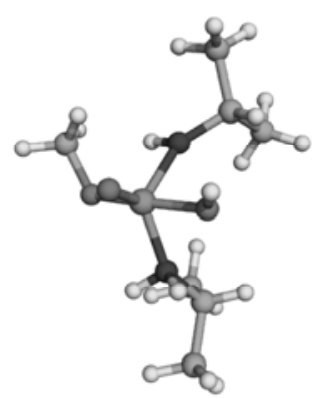

O8-P: 1.74 [1.73]

P-N4: 1.97 [1.93]

N4-H9: 1.02 [1.03]

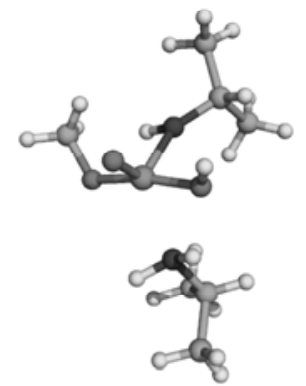

TSc2

O8-P: 1.71 [1.70]

P-N4: 2.21 [2.18]

N4-H9: 1.02 [1.03]
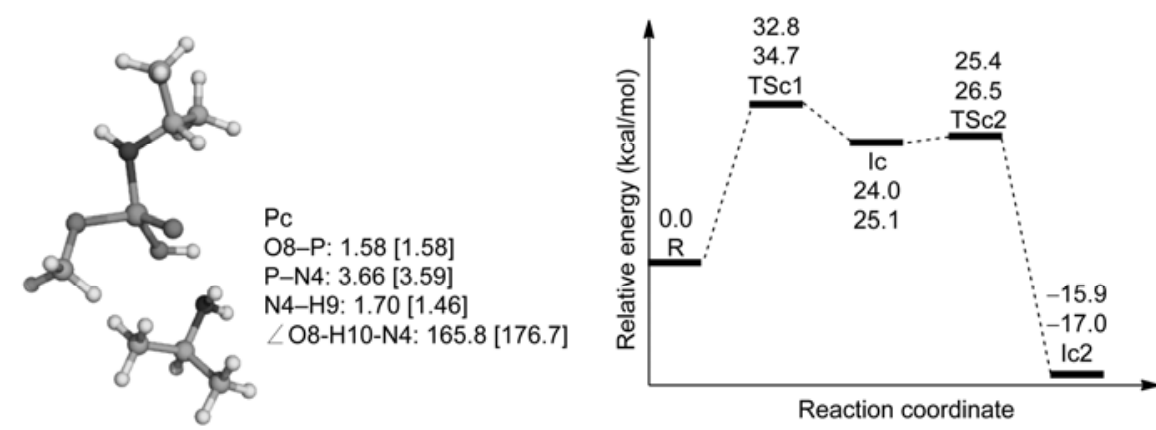

Figure 4 Optimized structures and the potential energy surface for the aging reaction. The values out of and in square brackets are for the gas phase and solvent phase, respectively. The units of distance, angle are angstrom, degree, respectively. 
isopropylamine group is released to form the final product (Pc) while the $\mathrm{P}-\mathrm{N} 4$ bond in Ic is broken. Obviously, this is a two-step addition-elimination mechanism, the same as the phosphonylation reaction.

$\mathrm{Rc}$ is stabilized through a weak hydrogen bond between the $\mathrm{H} 9$ atom of $\mathrm{H}_{2} \mathrm{O}$ and the $\mathrm{N} 4$ atom of the mipafox-enzyme conjugate, with a bond length of $2.02 \AA$ and a bond angle of $167.0^{\circ}$. The atom distance between $\mathrm{P}$ in the mipafox-enzyme conjugate and $\mathrm{O} 8$ in $\mathrm{H}_{2} \mathrm{O}$ is $3.92 \AA$, indicating that there is no interaction between the two separated species.

The transition state corresponding to hydride transfer and nucleophilic attack, TSc1, has only one imaginary frequency of $833 \mathrm{i} \mathrm{cm}^{-1}$. The vibrational mode corresponding to the imaginary frequency obviously indicates the direct nucleophilic attack by $\mathrm{O} 8$ in $\mathrm{H}_{2} \mathrm{O}$ on the $\mathrm{P}$ atom of the mipafoxenzyme conjugate. At the same time, the proton transfer from $\mathrm{H} 9$ to N4 occurs. The NPA charges calculated in the same theoretical level show that the attacking $\mathrm{O} 8$ in $\mathrm{H}_{2} \mathrm{O}$ has a negative charge of $-0.69 \mathrm{au}$, which is 0.25 au more than that in Rc, indicating a stronger nucleophilic character. The distance between the $\mathrm{P}$ and $\mathrm{O} 8$ atoms is $2.14 \AA$, and the bond length of P-N4 increases to $1.84 \AA$ from $1.71 \AA$ in Rc. The Gibbs free energy barrier of this step is $32.8 \mathrm{kcal} / \mathrm{mol}$ at $298 \mathrm{~K}$ and $1 \mathrm{~atm}$ pressure.

The intermediate Ic, formed through proton transfer and nucleophilic attack, has a trigonal bipyramidal structure similar to Ia and Ib1. It lies $24 \mathrm{kcal} / \mathrm{mol}$ above Rc, indicating that it is not as stable as Rc. The bond length of $\mathrm{P}-\mathrm{N} 4$ is weakened to be 1.97 from $1.71 \AA$ in Rc. Moreover, the $\mathrm{P}-\mathrm{O} 8$ bond is strengthened as its length is shortened to 1.74 from $2.14 \AA$ in TSc1.

The transition state (TSc2) for the elimination of a single isopropylamine group lies $1.4 \mathrm{kcal} / \mathrm{mol}$ above Ic. The one imaginary frequency is estimated as $120 \mathrm{i} \mathrm{cm}^{-1}$ and the vibrational mode shows the direct elimination of a single isopropylamine group. The atomic distance of $\mathrm{P}-\mathrm{N} 4$ is increased to 2.21 from $1.97 \AA$ in Ic, indicating that a weaker interaction may exist between the $\mathrm{P}$ atom and $\mathrm{N} 4$ atom.

The product $(\mathrm{Pc})$ lies $41.3 \mathrm{kcal} / \mathrm{mol}$ below TSc2 and 15.9 $\mathrm{kcal} / \mathrm{mol}$ below Rc, indicating that Pc is more stable than Rc. Two parts of Pc are connected by a weak N4-H10 hydrogen bond interaction, with a bond length of $1.70 \AA$ and a bond angle of $165.8^{\circ}$. The product $(\mathrm{Pc})$ is consistent with the ex- perimental results [12].

From the discussion above, it is clear that the aging reaction also employs a two-step addition-elimination mechanism with the addition as the rate-limiting step. This reaction is exothermic by $15.9 \mathrm{kcal} / \mathrm{mol}$ and hence, thermodynamically feasible. This result may provide a useful guide to study the aging mechanism of the AChE-mipafox adduct.

To evaluate the solvent effects on this reaction, the structures of reactant, transition states, intermediate, and product were optimized using the CPCM water model and the results are shown in Figure 4. The NPA charges of several important atoms involved in the solvent phase structures by NBO analysis are listed in Table 2 and the potential energy surface of the solvent phase pathway is shown in Figure 4. It is evident that the structures of all the stationary points are influenced by the solvent effect and differ from the relative structures in the gas phase. The charges of the $\mathrm{O} 8$ atom in both TSc2 and Pc and the charge of N4 in TSc2 in the solvent phase are not in agreement with them in the gas phase and this might be caused by use of the polar solvent. However, the free energy barrier of the rate-limiting step in the solvent phase is $1.9 \mathrm{kcal} / \mathrm{mol}$ higher than that in the gas phase indicating that the solvent only slightly influences the aging process.

\section{Conclusions}

Quantum chemical calculations were performed to theoretically investigate phosphonylation and aging mechanisms of butyrylcholinesterase with mipafox at the B3LYP/6-311G $(d, p)$ level of theory, and the potential energy surfaces of the phosphonylation reaction and aging reaction were identified. In the phosphonylation reaction, two different models (pathway A and pathway B) represent the situation with and without the effect of the catalytic triad of BChE, respectively. Both employ the same reaction pathway which is a two-step addition-elimination reaction with the addition (the first step) as the rate-limiting step. The differences between the two pathways reveal that the catalytic triad of $\mathrm{BChE}$ can effectively accelerate the phosphonylation process through the proton transfer. These results are consistent with the phosphonylation mechanism of sarin and AChE by Wang

Table 2 NPA charges of several important atoms involved in aging reaction by NBO analysis ${ }^{\text {a) }}$

\begin{tabular}{|c|c|c|c|c|c|}
\hline & $\mathrm{O} 1$ & O6 & O8 & $\mathrm{P}$ & $\mathrm{F}$ \\
\hline $\mathrm{Rc}$ & $-0.52[-0.52]$ & $-0.57[-0.57]$ & $-0.46[-0.46]$ & $1.24[1.24]$ & $-0.67[-0.67]$ \\
\hline $\mathrm{TSc} 1$ & $-0.58[-0.58]$ & $-0.57[-0.57]$ & $-0.69[-0.71]$ & 1.25 [1.24] & $-0.58[-0.56]$ \\
\hline Ic & $-0.62[-0.62]$ & $-0.59[-0.60]$ & $-0.64[-0.65]$ & $1.29[1.28]$ & $-0.50[-0.50]$ \\
\hline $\mathrm{TSc} 2$ & $-0.58[-0.59]$ & $-0.57[-0.60]$ & $-0.69[-0.58]$ & $1.25[1.28]$ & $-0.58[-0.51]$ \\
\hline
\end{tabular}

a) The values (au) out of and in square brackets are got in gas phase and in solvent phase, respectively. 
et al. [16]. However, the Gibbs free energy barriers of the present reaction are higher than that in the phosphonylation reaction of sarin and AChE by Wang et al. [16] because the negative charges around the $\mathrm{O} 1$ atom of the serine residue in the present study are less. Similar to the phosphonylation reaction, the aging process also employs a two-step addition-elimination reaction with the addition (the first step) as the rate-limiting step. This result may provide a useful guide for studying the aging mechanism of $\mathrm{AChE}$ and mipafox.

To evaluate the solvent effects, a CPCM model with a dielectric constant $\varepsilon=78.39$ was employed to perform the structure optimizations and IRC calculations at a B3LYP/6$311 \mathrm{G}(\mathrm{d}, \mathrm{p})$ level. The phosphonylation reaction is strongly influenced by the solvent effects, which lowers the Gibbs free energy barriers and increases the rate of the entire phosphonylation reaction. However, the solvent effects were negligible on the aging reaction.

This work was supported by the National Natural Science Foundation of China (20973077 and 20973049), the Program for New Century Excellent Talents in University (NCET), the Doctor Foundation by the Ministry of Education (20112303110005), the Foundation for the Department of Education of Heilongjiang Province (1152G010 and 11551077), and the Science Fundation for Leading Experts in Academe of Harbin of China (2011RFJGSO26).

1 Milatovic D, Dettbam W D. Modification of acetylcholinesterase during adaptation to chronic, subacute paraoxon application in rat. Toxicol Appl Pharmacol, 1996, 136: 20-28

2 Schetinger M R C, Porto N M, Moretto M B, et al. New benzodiazepines alter acetylcholinesterase and ATPDase activities. Neurochem Res, 2000, 25: 949-955

3 Massoulie J, Sussman J, Bon S, et al. Chapter 15: Structure and functions of acetylcholinesterase and butyrylcholinesterase. Prog Brain Res, 1993, 98: 139-146

4 Massoulie J, Pezzementi L, Bon S, et al. Molecular and cellular biology of cholinesterase. Prog Neurobiol, 1993, 41: 31-91

5 Xie W H, Stribley J A, Chatonnet A, et al. Postnatal developmental delay and supersensitivity to organophosphate in gene-targeted mice lacking acetylcholinesterase. J Pharmacol Exp Ther, 2000, 293: 869_ 902

6 Zhan C G, Zheng F, Landry D W, Fundamental reaction mechanism for cocaine hydrolysis in human butyrylcholinesterase. J Am Chem Soc, 2003, 125: 2462-2474

7 Sussman J L, Harel M, Frolow F, et al. Atomic structure of acetylcholinesterase from Torpedo California: A prototypic acetylecholine-binding protein. Science, 1991, 253: 872-879

8 Nicolet Y, Lockridge O, Masson P, et al. Crystal structure of human butyrylcholinesterase and of its complexes with substrate and products. J Biol Chem, 2003, 278: 41141-41147

9 Chhaliani V, Deer D, Earles B, et al. Purification and partial amino acid sequence analysis of human erythrocyte acetylcholinesterase. FEBS Lett, 1989, 247: 279-282
10 Peeples E S, Schopfer L M, Duysen E G, et al. Albumin, a new biomarker of organophosphorus toxicant exposure, identified by mass spectrometry. Toxicol Sci, 2005, 83: 303-312

11 Thompson C M, Richardson R J, Chichester U K. Pesticide Toxicology and International Regulation. Chichester: John Wiley \& Sons Ltd, 2004. 89-127

12 Kropp T J, Richardson R J. Mechanism of aging of mipafox-inhibited butyrylcholinesterase. Chem Res Toxical, 2007, 20: 504-510

13 Kropp T J, Richardson R J. Aging of mipafox-inhibited human acetylcholinesterase proceeds by displacement of both isopropylamine groups to yield a phosphate adduct. Chem Res Toxical, 2006, 19: 334-339

14 Zhang Y K, Kua J, Andrew McCammon J. Role of the catalytic triad and oxyanion hole in acetylcholinesterase catalysis: An ab initio QM/ MM study. J Am Chem Soc, 2002, 124: 10572-10577

15 Gao D Q, Zhan C G. Modeling evolution of hydrogen bonding and stabilization of transition states in the process of cocaine hydrolysis catalyzed by human butyrylcholinesterase. Proteins, 2006, 62: 99110

16 Wang J, Gu J D, Leszczynski J. Phosphonylation mechanisms of sarin and acetylcholinesterase: A model DFT study. J Phys Chem B, 2006, 110, 7567-7573

17 Wang J, Gu J D, Leszczynski J, et al. Oxime-induced reactivation of sarin-inhibited AChE: A theoretical mechanisms study. J Phys Chem B, 2007, 111: 2404-2408

18 Frisch M J, Trucks G W, Schlegel H B, et al. Gaussian Inc: Wallingford, CT, 2003

19 Becke A D. Density-functional thermochemistry. III. The role of exact exchange. J Chem Phys, 1993, 98: 5648-5652

20 Lee C, Yang W, Parr R G. Development of the Colle-Salvetti correlation-energy formula into a functional of the electron density. Phys Rev B, 1988, 37: 785-789

21 Miehlich B, Savin A, Stoll H, et al. Results obtained with the correlation energy density functional of becke and Lee, Yang and Parr. Chem Phys Lett, 1989, 157: 200-206

22 Hehre W J, Radom L, Schleyer P R, et al. Ab Initio Molecular Orbital Theory. New York: Wiley, 1986

23 Klamt A, Schuurmann G. COSMO_A new approach to dielectric screening in solvents with explicit expressions for the screening energy and its gradient. J Chem Soc Perkin Trans, 1993, 2: 799-805

24 Andzelm J, Kolmel C, Klamt A. Incorporation of solvent effects into density functional calculations of molecular energies and geometries. J Chem Phys, 1995, 103: 9312

25 Barone V, Cossi M. Quantum calculation of molecular energies and energy gradients in solution by a conductor solvent model. J Phys Chem A, 1998, 102: 1995-2001

26 Cossi M, Gega N, Scalmani G, et al. Energies, structures, and electronic properties of molecules in solution with the CPCM solvation model. J Comput Chem, 2003, 24: 669-681

27 Reed A E, Weinstock R B, Weinhold F. Natural population analysis. J Chem Phys, 1985, 83: 735

28 Reed A E, Weinhold F. Natural localized molecular orbitals. J Chem Phys, 1985, 83: 1736

29 Reed A E, Curtiss L A, Weinhold F. Intermolecular interactions from a natural bond orbital, donor-acceptor viewpoint. Chem Rev, 1988, 88: 899-926

30 Reed A E, Schleyer P R. Chemical bonding in hypervalent molecules. The dominance of ionic bonding and negative hyperconjugation over d-orbital participation. J Am Chem Soc, 1990, 112: 1434-1445

Open Access This article is distributed under the terms of the Creative Commons Attribution License which permits any use, distribution, and reproduction in any medium, provided the original author(s) and source are credited. 